\title{
Nursing Care Management of Chronic Myeloid Leukemia - A Case Report
}

\author{
Shakib Hasan Sheikh ${ }^{1}$, Vaishali Tembhare², Seema Singh ${ }^{3}$, Savita Pohekar ${ }^{4}$, Samruddhi Gujar ${ }^{5}$ \\ 1, 2, 3,4,5 Department of Medical-Surgical Nursing, Smt. Radhikabai Meghe Memorial College of Nursing, \\ Datta Meghe Institute of Medical Sciences, Sawangi (M), Wardha, Maharashtra, India.
}

\section{INTRODUCTION}

Excessive growth of mature granulocytes in the bone marrow induces chronic myeloid leukemia. The excess neoplastic granulocytes travel massively into the peripheral blood and in the end invade the liver and spleen. The protein encoded on the Philadelphia chromosome by the newly created $B C R-A B L$ gene interferes with normal cell cycle activities, including regulating cell proliferation. Philadelphia chromosome is present in 90 - 95 percent of chronic myeloid leukemia (CML) patients. Their involvement is often a vital indicator of persistent disease or posttreatment relapse.

However, for the diagnosis of CML, the presence of the Philadelphia chromosome is not specific since it is also present in acute lymphocytic leukemia (ALL) and rarely in acute myeloid leukemia (AML). ${ }^{1}$ Chronic myeloid leukemia is a myeloproliferative neoplasm (MPN) characterised by involvement of the fusion gene BCRABL1 located in the Philadelphia chromosome. In reactive neutrophilia or chronic neutrophilic leukemia, the Ph chromosome is pathognomonic to CML and is never registered.2,3

\section{PRESENTATION OF CASE}

A 65-years-old male patient who was apparently alright 2 months back was admitted to the hospital with the chief complaints of chest pain, shortness of breath, fatigue, malaise and weight loss for last 20 days. Peripheral blood evaluation and bone marrow examination were done. In peripheral blood examination, RBC was low (2.5 $\mathrm{m} /$ cumm), HCT was decreased, $34.9 \%$, platelet count was low 50,000 and WBC was high 27,000 cumm. Leukocytosis with severe neutrophilia was 90 percent marked in the peripheral smear with increased cell granulation. Basophils rose by $8 \%$, and there were no blasts in circulation. In bone marrow analysis the chronic myeloid leukaemia was diagnosed as hypercellular with an expansion of the granulopoiesis population and marked neutrophilic proliferation based on investigations.

\section{NURSING INTERVENTIONS}

The patient received imatinib, which was modified to nilotinib $300 \mathrm{mg}$ PO bid due to imatinib intolerance. Thrombocytopenia (65 x 109 cells / L) developed a month later, and the dosage of nilotinib was reduced to $200 \mathrm{mg}$ of PO.

Patient was susceptible to infection due to inadequate primary defences. Hence,

- The patient was placed in a private room. Visitors were restricted as stated. Flowers and plants were restricted. Medical treatment was coordinated such that leukemic patients do not come into contact with workers who also care for respiratory or infectious disease patients.

- A good hand washing protocol was followed for all staff and visitors.

- Temperature was monitored very closely.

- Tepid sponge bath was given.
Corresponding Author Dr. Shakib H. Sheikh,

Department of Medical-Surgical Nursing, Smt. Radhikabai Meghe Memorial College of Nursing, Datta Meghe Institute of Medical Sciences, Sawangi (M), Wardha,

Maharashtra, India.

E-mail: shakibsheikh122@gmail.com

DOI: $10.14260 /$ jemds/2021/252

How to Cite This Article:

Sheikh SH, Tembhare V, Singh S, et al. Nursing care management of chronic myeloid leukemia - a case report. J Evolution Med Dent Sci 2021;10(16):11821184, DOI: 10.14260/jemds/2021/252

Submission 24-09-2020,

Peer Review 18-11-2020,

Acceptance 24-11-2020,

Published 19-04-2021.

Copyright (c) 2021 Shakib Hasan Sheikh et al. This is an open access article distributed under Creative Commons Attribution License [Attribution 4.0 International (CC BY 4.0)] 
Patient had risk of fluid volume deficiency secondary to decreased fluid intake, e.g., nausea and anorexia. Hence,

- Intake \& output, and fluid balance was monitored. Note: There was reduced output of urine in the presence of adequate intake. The $\mathrm{pH}$ of particular gravity and urine was measured.

- Daily weight of the patient was recorded.

- Excellent nutrition was promoted.

- $\quad$ Blood pressure and heart rate was monitored.

- $\quad$ Patient was encouraged to consume 3 - $4 \mathrm{~L}$ / day of fluids when resuming oral intake.

Patient had acute pain when administered with physical agents like bone marrow packed with leukemic cells. Hence,

- Pain was investigated and reported. The degree and changes in site were noted.

- Vital signs, nonverbal signs of stress in the muscles and restlessness were recorded.

- A quiet atmosphere was maintained, and stressful impulses were reduced. Noise restriction or elimination, illumination and frequent interruptions was avoided.

- Joints, tips with pillows or padding in a position of comfort and support was given.

- $\quad$ Periodic reposition and support with gentle exercises was provided.

The nursing task is particularly difficult during the chronic phase of myeloid leukaemia because there are many physical and psychosocial needs of the patient. The diagnosis of leukaemia, as with other types of cancers, can cause great fear and result in death. With many painful and undesirable effects, it can be regarded as a hopeless disease. ${ }^{4}$ Many nurses are involved in ${ }^{1}$ upgrading the physical working of patients, ${ }^{2}$ instructing patients that intense symptoms are commonly transitory and ${ }^{3}$ engaging patients to address their satisfaction issues.

Ambulatory care: the patient with chronic myeloid leukaemia requires continuous treatment to monitor signs and symptoms of disease control or relapse. For long-term survivors of chronic myeloid leukaemia, recovery aims to control the physical, psychological and social consequences and delayed effects of the condition and its treatment. Exploring community resources can reduce both the financial burden and the feelings of dependence. Often, spiritual support services are provided. ${ }^{5}$

Vigilant follow-up treatment helps to guarantee the particular needs of the cancer patient are identified and addressed. Such needs may also require a referral or consultation. For example, an exercise regimen to avoid posttreatment deficits caused by drug-induced peripheral neuropathy can be handled by physical therapy workers. ${ }^{6}$

\section{Nutrition Management for Chronic Myeloid Leukemia}

Eating a healthy diet during and after CML treatment can help maintain a healthy weight and support immune system. ${ }^{7}$

The Leukemia \& Lymphoma Society suggests a healthy diet to help the body recover. Five to ten fruit and vegetable servings, whole cereals and pulses, low-fat foods that are high in protein, such as fish, poultry, lean meat and low-fat dairy must be consumed.

Ideally, one of daily vegetable servings should be a cruciferous vegetable. For example, kale, brassica, sprouts, cabbage, watercress and palak.

\section{DISCUSSION}

This case study of CML was complicated by diabetes mellitus and hypertension diseases in an elderly patient. Busulfan was the first drug used to bring haematological remission.

Hydroxyurea is similarly active but less mutagenic, and the toxicity profile is more favourable than busulfan. Interferon can cause haematological remission and can remove positive clones from Philadelphia when evaluated by classical cytogenetics. This medicine is also used with cytarabine in conjunction. ${ }^{8}$

In a region known as micro- $B C R$, CML-N has a $B C R$ breakpoint on chromosome 22 that occurs downstream of $\mathrm{M}$ BCR between exons 17 and 20 and is encoded with a larger fusion protein, p2300.9 When present and sub-typed by reverse transcription-polymerase chain reaction, translocation can be detected using FISH analysis.

A pan-sample detecting the three known breakpoints for the $\mathrm{Ph}$ chromosome, one of which is micro- $B C R$, is the FISH probe in our case. RT-PCR sub-typing measures the sum of the two types of BCR-ABL fusion transcripts, p210 and p190, respectively, seen with the Ph chromosome in CML and acute lymphoblastic leukaemia. ${ }^{10}$

Financial or other competing interests: None.

Disclosure forms provided by the authors are available with the full text of this article at jemds.com.

The patients' informed consent was taken and signed by the patient before writing a case report.

\section{REFERENCES}

[1] Chintamani, Mani M. Lewis's Medical-surgical nursing. Third South Asia Edition. 3rd edn. [Cited 2020 Jun 11]. https://www.elsevier.com/books/lewiss-medicalsurgical-nursing-third-south-asiaedition/chintamani/978-81-312-5331-1

[2] Vardiman JW, Melo JV, Baccarani M, et al. Chronic myelogenous leukemia, BCR-ABL1+. In: Swerdlow SH, Campo E, Harris NL, et al, eds. WHO classification of tumors of hematopoietic and lymphoid tissues. Vol. 2. $4^{\text {th }}$ edn. Lyon, France: IARC Press 2008:32-7.

[3] Pane F, Frigeri F, Sindona M, et al. Neutrophilic-chronic myeloid leukemia: a distinct disease with a specific molecular marker (BCR/ABL with C3/A2 junction). Blood 1996;88(7):2410-4.

[4] Aigner KR, Stephens FO. Basics of Oncology. Berlin, Heidelberg: Springer Berlin Heidelberg 2009. http://link.springer.com/10.1007/978-3-540-92925-3

[5] Smeltzer SC, Bare BG. Brunner \& Suddarth's Textbook of medical-surgical nursing. $10^{\text {th }}$ edn. Philadelphia: Lippincott Williams \& Wilkins 2003. 
[6] Black JM, Hawks JH. Medical-surgical nursing clinical management for positive outcomes. $8^{\text {th }}$ edn. Vol. 1. USA: Elsevier 2008.

[7] Nutrition tips for Chronic Myeloid Leukemia. (CML) Healthline 2015 [cited 2021 Jan 5]. https://www.healthline.com/health/chronic-myeloidleukemia/nutrition-guide-for-cml
[8] Zafar MZ. Patient with chronic myeloid leukemia: a case study. J Cancer Sci Ther 2017;9(9):635-6.

[9] Saglio G, Guerrasio A, Rosso C, et al. New type of BCR/ABL junction in Philadelphia chromosome-positive chronic myelogenous leukemia. Blood 1990;76(9):1819-24.

[10] Melo JV. The diversity of BCR-ABL fusion proteins and their relationship to leukemia phenotype. Blood 1996;88(7):2375-84. 